\title{
Effect of multiple scattering on broadening and the frequency shift of spectral lines
}

\author{
S. Datta, S. Roy, ${ }^{*}$ and M. Roy \\ Physics and Applied Mathematics Unit, Indian Statistical Institute, 203 Barrackpur Trunk Road, Calcutta 700 035, India \\ M. Moles ${ }^{\dagger}$ \\ Instituto de Matemáticas y Física Fundamental, CSIC C/Serrano 123, 28006 Madrid, Spain \\ and Observatorio Astronómico Nacional, Apartado 1143, 28800 Alcalá de Henares, Spain
}

(Received 10 November 1997)

\begin{abstract}
The effect of multiple scattering on the broadening and the shift of the spectral lines of light propagating in a medium is investigated within Wolf's framework. The condition for no blueshift is derived. It is shown that under the same hypothesis the property $\Delta \omega_{N+1} \gg \delta_{N}$ is satisfied. Moreover, the no blueshift condition has been found to be closely related to the no blurring condition. These results might be of relevance in the astronomical domain. [S1050-2947(98)01107-X]
\end{abstract}

PACS number(s): 32.70.Jz

In a series of published papers over the last two decades, it was shown that the spectrum of light changes on propagation [1]. Source correlation and random media are shown to be responsible for it. The randomness in this framework is due to the fluctuating response function of the medium, e.g., its dielectric susceptibility. In our present work we use this framework assuming the presence of a large number of scatterers [2] to find the effect of multiple scattering on the broadening and frequency shift of the spectral lines: a question that could be important in the astronomical context.

First we briefly state the main results of Wolf's scattering mechanism. Let us consider a polychromatic field of light of central frequency $\omega_{0}$ and of width $\delta_{0}$, incident on the scatterer. The incident spectrum is of the form

$$
S^{(i)}(\omega)=A_{0} e^{\left[-\left(1 / 2 \delta_{0}^{2}\right)\left(\omega-\omega_{0}\right)^{2}\right]} .
$$

The spectrum of the scattered field is given by [3]

$$
S^{(\infty)}\left(\omega^{\prime}\right)=A \omega^{\prime 4} \int_{-\infty}^{\infty} K\left(\omega, \omega^{\prime}\right) S^{(i)}(\omega) d \omega
$$

which is valid within the first-order Born approximation [4]. Here $K\left(\omega, \omega^{\prime}\right)$ is the so-called scattering kernel and it plays the most important role in this mechanism. Instead of studying $\mathcal{K}\left(\omega, \omega^{\prime}\right)$ in detail, we consider a particular case supposing the correlation function $G(\vec{R}, T ; \omega)$ of the generalized dielectric susceptibility $\eta(\vec{r}, t ; \omega)$ of the medium is characterized by an anisotropic Gaussian function

$$
\begin{aligned}
G(\vec{R}, T ; \omega) & =\left\langle\eta^{*}(\vec{r}+\vec{R}, t+T ; \omega) \eta(\vec{r}, t ; \omega)\right\rangle \\
& =G_{0} \exp \left[-\frac{1}{2}\left(\frac{X^{2}}{\sigma_{x}^{2}}+\frac{Y^{2}}{\sigma_{y}^{2}}+\frac{Z^{2}}{\sigma_{z}^{2}}+\frac{c^{2} T^{2}}{\sigma_{\tau}^{2}}\right)\right] .
\end{aligned}
$$

\footnotetext{
*Electronic address: sisir@isical.ac.in

†Electronic address: moles@oan.es
}

Here $G_{0}$ is a positive constant, $\vec{R}=(X, Y, Z)$, and $\sigma_{x}, \sigma_{y}, \sigma_{z}, \sigma_{\tau}$ are correlation lengths [5]. $\mathcal{K}\left(\omega, \omega^{\prime}\right)$ can be obtained from the four-dimensional Fourier transform of the correlation function $G(\vec{R}, T ; \omega)$. In this case $\mathcal{K}\left(\omega, \omega^{\prime}\right)$ was shown to be of the form

$$
\mathcal{K}\left(\omega, \omega^{\prime}\right)=B \exp \left[-\frac{1}{2}\left(\alpha^{\prime} \omega^{\prime 2}-2 \beta \omega \omega^{\prime}+\alpha \omega^{2}\right)\right],
$$

where

$$
\begin{gathered}
\alpha=\frac{\sigma_{x}^{2}}{c^{2}} u_{x}^{2}+\frac{\sigma_{y}^{2}}{c^{2}} u_{y}^{2}+\frac{\sigma_{z}^{2}}{c^{2}} u_{z}^{2}+\frac{\sigma_{\tau}^{2}}{c^{2}} \\
\alpha^{\prime}=\frac{\sigma_{x}^{2}}{c^{2}} u_{x}^{\prime 2}+\frac{\sigma_{y}^{2}}{c^{2}} u_{y}^{\prime 2}+\frac{\sigma_{z}^{2}}{c^{2}} u_{z}^{\prime 2}+\frac{\sigma_{\tau}^{2}}{c^{2}} \\
\beta=\frac{\sigma_{x}^{2}}{c^{2}} u_{x} u_{x}^{\prime}+\frac{\sigma_{y}^{2}}{c^{2}} u_{y} u_{y}^{\prime}+\frac{\sigma_{z}^{2}}{c^{2}} u_{z} u_{z}^{\prime}+\frac{\sigma_{\tau}^{2}}{c^{2}} .
\end{gathered}
$$

Here $\hat{u}=\left(u_{x}, u_{y}, u_{z}\right)$ and $\hat{u}^{\prime}=\left(u_{x}^{\prime}, u_{y}^{\prime}, u_{z}^{\prime}\right)$ are the unit vectors in the directions of the incident and scattered fields, respectively.

Substituting Eqs. (1) and (4) in Eq. (2), we finally get

$$
S^{(\infty)}\left(\omega^{\prime}\right)=A^{\prime} e^{\left[-\left(1 / 2 \delta_{0}^{\prime 2}\right)\left(\omega^{\prime}-\bar{\omega}_{0}\right)^{2}\right]},
$$

where

$$
\begin{aligned}
& \bar{\omega}_{0}=\frac{|\beta| \omega_{0}}{\alpha^{\prime}+\delta_{0}^{2}\left(\alpha \alpha^{\prime}-\beta^{2}\right)}, \\
& \delta_{0}^{\prime 2}=\frac{\alpha \delta_{0}^{2}+1}{\alpha^{\prime}+\delta_{0}^{2}\left(\alpha \alpha^{\prime}-\beta^{2}\right)},
\end{aligned}
$$

$$
A^{\prime}=\sqrt{\frac{\pi}{2\left(\alpha \delta_{0}^{2}+1\right)}} A B A_{0} \omega_{0}^{\prime 4} \delta_{0} \exp \left[\frac{|\beta| \omega_{0} \bar{\omega}_{0}-\alpha \omega_{0}^{2}}{2\left(\alpha \delta_{0}^{2}+1\right)}\right] .
$$


Though $A^{\prime}$ depends on $\omega^{\prime}$, it was approximated by James and Wolf [3] to be a constant so that $S^{(\infty)}\left(\omega^{\prime}\right)$ can be considered to be Gaussian.

The relative frequency shift is defined as

$$
z=\frac{\omega_{0}-\bar{\omega}_{0}}{\bar{\omega}_{0}},
$$

where $\omega_{0}$ and $\bar{\omega}_{0}$ denote the unshifted and shifted central frequencies respectively. We say that the spectrum is redshifted or blueshifted according to whether $z>0$ or $z<0$, respectively. Here

$$
z=\frac{\alpha^{\prime}+\delta_{0}^{2}\left(\alpha \alpha^{\prime}-\beta^{2}\right)}{|\beta|}-1 .
$$

It is important to note that this $z$ number does not depend on the incident frequency, $\omega_{0}$. This is a very important aspect if the mechanism is to be considered in the astronomical domain. Expression (9) implies that the spectrum can be shifted to the blue or to the red, according to the sign of the term $\alpha^{\prime}+\delta_{0}^{2}\left(\alpha \alpha^{\prime}-\beta^{2}\right)>|\beta|$. To obtain the no-blueshift condition, we use Schwarz's inequality which implies that $\alpha \alpha^{\prime}$ $-\beta^{2} \geqslant 0$. Thus we can take

$$
\alpha^{\prime}>|\beta|
$$

as the sufficient condition to have only redshift by that mechanism.

Let us now assume that the light in its journey encounters many such scatterers. What we observe at the end is the light scattered many times, with an effect like that stated above in every individual process. Let there be $N$ scatterers between the source and the observer and $z_{n}$ denote the relative frequency shift after $n$th scattering of the incident light from the $(n-1)$ th scatterer, with $\omega_{n}$ and $\omega_{n-1}$ being the central frequencies of the incident spectra at $n$th and $(n-1)$ th scatterers. Then by definition,

$$
z_{n}=\frac{\omega_{n-1}-\omega_{n}}{\omega_{n}}, \quad n=1,2, \ldots, N
$$

or

$$
\frac{\omega_{n-1}}{\omega_{n}}=1+z_{n}, \quad n=1,2, \ldots, N
$$

Taking product over $n$ from $n=1$ to $n=N$, we get

$$
\frac{\omega_{0}}{\omega_{N}}=\left(1+z_{1}\right)\left(1+z_{2}\right) \cdots\left(1+z_{N}\right) .
$$

But the left hand side of the above equation is nothing but the ratio of the source frequency and the final or the observed frequency $z_{f}$. Hence,

$$
1+z_{f}=\left(1+z_{1}\right)\left(1+z_{2}\right) \cdots\left(1+z_{N}\right) .
$$

Since the $z$ number due to such effect does not depend upon the central frequency of the incident spectrum, each $z_{i}$ depends on $\delta_{i-1}$ only, not $\omega_{i-1}$ [here $\omega_{j}$ and $\delta_{j}$ denote the central frequency and the width of the incident spectrum at $(j+1)$ th scatterer]. To find the exact dependence we first find the broadening of the spectrum after $N$ number of scatterings.

Clearly, from Eq. (7),

$$
\begin{aligned}
\delta_{n+1}^{2} & =\frac{\alpha \delta_{n}^{2}+1}{\alpha^{\prime}+\left(\alpha \alpha^{\prime}-\beta^{2}\right) \delta_{n}^{2}} \\
& =\left(\frac{\alpha \delta_{n}^{2}+1}{\alpha^{\prime}}\right)\left[1+\delta_{n}^{2}\left(\frac{\alpha \alpha^{\prime}-\beta^{2}}{\alpha^{\prime}}\right)\right]^{-1} .
\end{aligned}
$$

From Eq. (7), we can write

$$
\omega_{N+1}=\frac{\omega_{N}|\beta|}{\alpha^{\prime}+\left(\alpha \alpha^{\prime}-\beta^{2}\right) \delta_{N}^{2}} .
$$

Then from Eqs. (8) and (9), we can write

$$
\begin{aligned}
z_{n+1} & =\frac{\omega_{n}-\omega_{n+1}}{\omega_{n+1}} \\
& =\frac{\alpha^{\prime}+\left(\alpha \alpha^{\prime}-\beta^{2}\right) \delta_{n}^{2}}{|\beta|}-1 \\
& =\frac{\alpha^{\prime}}{|\beta|}\left\{1+\left(\frac{\alpha \alpha^{\prime}-\beta^{2}}{\alpha^{\prime}}\right) \delta_{n}^{2}\right\}-1 .
\end{aligned}
$$

Let us assume that the redshift per scattering process is very small, i.e.,

$$
0<\epsilon=z_{n+1} \ll 1
$$

Then,

$$
1+\epsilon=\frac{\alpha^{\prime}}{|\beta|}\left\{1+\left(\frac{\alpha \alpha^{\prime}-\beta^{2}}{\alpha^{\prime}}\right) \delta_{n}^{2}\right\}
$$

or

$$
(1+\epsilon) \frac{|\beta|}{\alpha^{\prime}}=1+\left(\frac{\alpha \alpha^{\prime}-\beta^{2}}{\alpha^{\prime}}\right) \delta_{n}^{2} .
$$

In order to satisfy this condition under the sufficient condition for having only redshift, we see that the first factor in the right term cannot be much bigger than 1 , and, more important,

$$
\left(\frac{\alpha \alpha^{\prime}-\beta^{2}}{\alpha^{\prime}}\right) \delta_{n}^{2} \ll 1 .
$$

In that case, from Eq. (11), after neglecting higher-order terms, the expression for $\delta_{n+1}^{2}$ can be well approximated as

$$
\delta_{n+1}^{2} \approx\left(\frac{\alpha \delta_{n}^{2}+1}{\alpha^{\prime}}\right)\left[1-\delta_{n}^{2}\left(\frac{\alpha \alpha^{\prime}-\beta^{2}}{\alpha^{\prime}}\right)\right],
$$

which, after simplification, gives a very important recurrence relation: 


$$
\delta_{n+1}^{2}=\frac{1}{\alpha^{\prime}}+\frac{\beta^{2}}{\alpha^{\prime 2}} \delta_{n}^{2}
$$

Therefore

$$
\begin{aligned}
\delta_{n+1}^{2} & =\frac{1}{\alpha^{\prime}}+\frac{\beta^{2}}{\alpha^{\prime 2}} \delta_{n}^{2} \\
& =\frac{1}{\alpha^{\prime}}+\frac{\beta^{2}}{\alpha^{\prime 2}}\left[\frac{1}{\alpha^{\prime}}+\frac{\beta^{2}}{\alpha^{\prime 2}} \delta_{n-1}^{2}\right] \\
& =\left(\frac{\beta^{2}}{\alpha^{\prime 2}}\right)^{2} \delta_{n-1}^{2}+\frac{1}{\alpha^{\prime}}\left(1+\frac{\beta^{2}}{\alpha^{\prime 2}}\right) \cdots \\
& =\left(\frac{\beta^{2}}{\alpha^{\prime 2}}\right)^{n+1} \delta_{0}^{2}+\frac{1}{\alpha^{\prime}}\left(1+\frac{\beta^{2}}{\alpha^{\prime 2}}+\cdots+\frac{\beta^{2 n}}{\alpha^{\prime 2 n}}\right) .
\end{aligned}
$$

Thus

$$
\delta_{N+1}^{2}=\left(\frac{\beta^{2}}{\alpha^{\prime 2}}\right)^{N+1} \delta_{0}^{2}+\frac{1}{\alpha^{\prime}}\left(1+\frac{\beta^{2}}{\alpha^{\prime 2}}+\cdots+\frac{\beta^{2 N}}{\alpha^{\prime 2 N}}\right) .
$$

As the number of scatterings increases, the width of the spectrum obviously increases and the most important topic to be considered is whether this width is below the tolerance limit or not, from the observational point of view. There may be several measures of that tolerance limit. One of them is the sharpness ratio, defined as

$$
Q=\frac{\omega_{f}}{\delta_{f}}
$$

where $\omega_{f}$ and $\delta_{f}$ are the mean frequency and the width of the observed spectrum.

After $N$ number of scatterings, this sharpness ratio, say $Q_{N}$, is given by the following recurrence relation:

$$
Q_{N+1}=Q_{N} \sqrt{\frac{\alpha^{\prime}}{\alpha^{\prime}+\left(\alpha \alpha^{\prime}-\beta^{2}\right) \delta_{N}^{2}}-\frac{1}{\alpha \delta_{N}^{2}+1}} .
$$

It is easy to verify that the expression under the square root lies between 0 and 1 . Therefore $Q_{N+1}<Q_{N}$, and the line is broadened as the scattering process goes on.

Now let us consider the following two conditions.

Case I. Broadening under the sufficient condition for redshift. Let $|\beta|<\alpha^{\prime}$. Then the series in Eq. (16) converges to a finite sum. For large $N$,

$$
\delta_{N}^{2} \approx \frac{\alpha^{\prime}}{\alpha^{\prime 2}-\beta^{2}}
$$

Thus the effective broadening converges to a constant value which is independent of the initial distribution (i.e., of the source). It signifies that the spectrum of light from any source suffers from the same limiting broadening which is entirely determined by the medium.

Now,

$$
\begin{aligned}
\Delta \omega_{N+1} & =\omega_{0}-\omega_{N+1} \\
& =\omega_{0}-\frac{\omega_{N}|\beta|}{\alpha^{\prime}+\left(\alpha \alpha^{\prime}-\beta^{2}\right) \delta_{N}^{2}} \\
& =\omega_{0}\left[1-m \frac{|\beta|}{\alpha^{\prime}+\left(\alpha \alpha^{\prime}-\beta^{2}\right) \delta_{N}^{2}}\right],
\end{aligned}
$$

where $m=\omega_{N} / \omega_{0}=1 /\left(1+z_{f}\right)$. Then,

$$
1-\frac{\Delta \omega_{N+1}}{\omega_{0}}=m \frac{|\beta|}{\alpha^{\prime}+\left(\alpha \alpha^{\prime}-\beta^{2}\right) \delta_{N}^{2}}
$$

or

$$
\left(\alpha \alpha^{\prime}-\beta^{2}\right) \delta_{N}^{2}\left[1-\frac{\Delta \omega_{N+1}}{\omega_{0}}\right]-\alpha^{\prime} \frac{\Delta \omega_{N+1}}{\omega_{0}}=m|\beta|-\alpha^{\prime}
$$

or

$$
\alpha^{\prime} \frac{\Delta \omega_{N+1}}{\omega_{0}}-\left(\alpha \alpha^{\prime}-\beta^{2}\right) \delta_{N}^{2}\left[1-\frac{\Delta \omega_{N+1}}{\omega_{0}}\right]>0,
$$

since $m<1$, and therefore $m|\beta|-\alpha^{\prime}<0$. From this relation we can claim that

$$
\Delta \omega_{N+1} \gg \delta_{N}
$$

if the following condition holds:

$$
\frac{\delta_{N} \omega_{0}\left(\alpha \alpha^{\prime}-\beta^{2}\right)}{\alpha^{\prime}+\left(\alpha \alpha^{\prime}-\beta^{2}\right) \delta_{N}^{2}} \gg 1 .
$$

The above result may also be verified from the observational data [3].

Case II. Let $|\beta|>\alpha^{\prime}$. In this case, for $\delta_{0}>0$, the spectrum may either be redshifted or be blueshifted according to Eq. (9). But from Eq. (16) we see that $\delta_{N}^{2}$, in this case, becomes unbounded with respect to $N$. Therefore, for large $N$, the effective broadening of the spectrum becomes so large that no distinct line can be observed. Only a blurred image will be formed from which no conclusion can be drawn [6].

Thus we can take the second case as the blurring condition of the spectra. This type of broadening is unavoidable since it depends only on the characteristics of the medium through which the light propagates. It is important to note that under this circumstance we can neither find the frequency shift nor even conclude whether the spectrum is redshifted or blueshifted. This situation turns out to be very interesting when we study the astrophysical observations, particularly the quasar spectra. When we observe a spectrum, due to the result (17), the shifts of the prominent lines dominate over the effective broadening. Also the observed spectra are strongly redshifted which can easily be seen from case I mentioned above. And, the most important fact is that even if there exist some blueshifts, they cannot be observed, by case 
II. Thus we can predict that whenever a sharp spectrum is observed, it is found to be redshifted-exactly matching with the experimental observations. Thus the no-blueshift condition is very closely related to the no blurring condition.
M.M. acknowledges financial support by DGICYT (Spain); Grant No. PB93-0139. One of the authors (S.R.) is thankful to CSIC (Spain) for hospitality and support during the time when part of this work was done.
[1] D. F. V. James and E. Wolf, Rep. Prog. Phys. 59, 771 (1996).

[2] S. Datta, S. Roy, M. Roy, and M. Moles, Int. J. Theor. Phys. (to be published).

[3] D. F. V. James and E. Wolf, Phys. Lett. A 146, 167 (1990).
[4] E. Wolf and M. Born, Principles of Optics, 6th ed. (Pergamon, Oxford, England, 1980).

[5] L. Mandel and E. Wolf, Opt. Commun. 8, 95 (1973).

[6] E. Wolf, NPL Technical Bulletin, New Delhi, October, 1991. 